\title{
Prognostic value of the immunohistochemistry markers CD56, TTF-1, synaptophysin, CEA, EMA and NSE in surgically resected lung carcinoid tumors
}

\author{
VASILIKI EPAMEINONDAS GEORGAKOPOULOU ${ }^{1}$, ELEFTHERIOS ZYGOURIS ${ }^{2}$, \\ CHRISTOS DAMASKOS $^{3,4}$, AIKATERINI PIERRAKOU ${ }^{5}$, PETROS PAPALEXIS ${ }^{6,7}$, NIKOLAOS GARMPIS ${ }^{4,8}$, \\ AIKATERINI ARAVANTINOU-FATOROU ${ }^{9}$, SERAFEIM CHLAPOUTAKIS ${ }^{10}$, EVANGELOS DIAMANTIS ${ }^{11}$, \\ CHRISTOS NIKOKIRIS $^{12}$, AIKATERINI GKOUFA ${ }^{9}$, PAGONA SKLAPANI ${ }^{13}$, NIKOLAOS TRAKAS ${ }^{14}$, \\ JIM JANINIS $^{2}$, DEMETRIOS A. SPANDIDOS ${ }^{15}$ and JUBRAIL DAHABREH ${ }^{2}$
}

\begin{abstract}
${ }^{1}$ Pulmonology Department, Laiko General Hospital, Athens $11527 ;{ }^{2}$ Oncology Department, Athens Medical Group, Athens 15125; ${ }^{3}$ Renal Transplantation Unit, Laiko General Hospital; ${ }^{4}$ N.S. Christeas Laboratory of Experimental Surgery and Surgical Research, Medical School, National and Kapodistrian University of Athens, Athens 11527; ${ }^{5}$ Department of Pathology, Athens Medical Group, Athens 15125; ${ }^{6}$ First Department of Propedeutic Internal Medicine, Laiko General Hospital, Medical School, National and Kapodistrian University of Athens, Athens 11527; ${ }^{7}$ Department of Biomedical Sciences, University of West Attica, Athens $12243 ;{ }^{8}$ Second Department of Propedeutic Surgery, Laiko General Hospital, Medical School, National and Kapodistrian University of Athens; ${ }^{9}$ First Department of Internal Medicine, Laiko General Hospital, Athens 11527; ${ }^{10}$ Department of Thoracic Surgery, Agios Savvas Hospital, Athens 11522; ${ }^{11}$ Unit of Endocrinology and Diabetes Center, Athens Hospital G. Gennimatas, Athens 11527; ${ }^{12}$ Intensive Care Unit, Sismanogleio Hospital, Athens 15126;

${ }^{13}$ Department of Cytology, Mitera Hospital, Athens 15123; ${ }^{14}$ Department of Biochemistry, Sismanogleio Hospital, Athens $15126 ;{ }^{15}$ Laboratory of Clinical Virology, School of Medicine, University of Crete, 71003 Heraklion, Greece
\end{abstract}

Received October 12, 2021; Accepted November 24, 2021

DOI: $10.3892 / \mathrm{mco} .2021 .2464$

\begin{abstract}
Lung carcinoid tumor is a type of neuroendocrine tumor, which is subdivided into typical carcinoid (TC) and atypical carcinoid (AT), based on the rate of mitosis and the presence of necrosis. Several prognostic factors for lung carcinoids have been reported in the literature, including the type, Ki67 index, stage, chemotherapy and radiation therapy. In the present study, 108 cases with resected carcinoid lung tumors were enrolled and the expression of CD56, thyroid transcription factor 1, synaptophysin, carcinoembryonic antigen, epithelial membrane antigen and neuron-specific enolase (NSE) in the resected tissue specimens was immunohistochemically analyzed. Patients with positive staining for NSE had an unfavorable survival prognosis compared with patients with
\end{abstract}

Correspondence to: Dr Petros Papalexis, First Department of Propedeutic Internal Medicine, Laiko General Hospital, Medical School, National and Kapodistrian University of Athens, 17 Agiou Thoma Street, Athens 11527, Greece

E-mail: petranpapalex@gmail.com

Key words: pulmonary carcinoids, thyroid transcription factor 1, synaptophysin, neuron-specificenolase, immunohistochemistry markers negative staining for NSE (137.2 vs. 150.0 months, $\mathrm{P}=0.044$ ). According to univariate analysis, none of the above immunohistochemistry markers was associated with survival, and according to multivariate analysis, NSE was an independent influencing factor for survival inpatients with AT $(\mathrm{P}=0.046)$ and furthermore, the stage was an independent factor of survival in patients with $\mathrm{TC}(\mathrm{P}=0.005)$.

\section{Introduction}

Lung carcinoid tumors are uncommon neuroendocrine malignant tumors, accounting for $<1 \%$ of all lung cancers. There are two subcategories: Typical carcinoids (TC), which are low-grade neuroendocrine tumors, and atypical carcinoids (AC), which are intermediate-grade neuroendocrine tumors. The majority of pulmonary carcinoids are observed in the center of the lung and have the ability to metastasize $(1,2)$.

The mean age at diagnosis is $\sim 50$ years but lung carcinoid tumors may occur at a wide range of ages (5-90 years). In addition, they are the most frequent type of childhood primary lung tumor. Risk factors for carcinoid development include a family history of carcinoids and being a carrier of the multiple endocrine neoplasia type 1 gene, while tobacco smoking has been correlated with the pathogenesis of atypical carcinoids to a much lesser extent than small cell or large cell neuroendocrine carcinomas $(3,4)$. 
The categorization into TCs and ACs is based on the rate of mitosis and the presence of necrosis. Immunohistochemistry is essential for diagnosis, with an antibody panel consisting of chromogranin A, synaptophysin and CD56 being recommended $(5,6)$. In addition, certain carcinoids exhibit staining for thyroid transcription factor 1 (TTF-1). The Ki67 labeling index is utilized in order to avoid misdiagnosis of high-grade neuroendocrine carcinomas $(5,6)$. Furthermore, neuron-specific enolase (NSE) is a highly specific marker for neuroendocrine cells and is expressed in lung carcinoids (7). In certain cases of lung carcinoids, positive reactions for epithelial membrane antigen (EMA) and carcinoembryonic antigen (CEA) have been described $(8,9)$.

Several prognostic factors for pulmonary carcinoids have been reported, including age, sex, tumor size, stage, type, type of surgical procedure performed, Ki67 expression, chemotherapy and radiation therapy $(10,11)$. CD56, TTF-1, synaptophysin, CEA, EMA and NSE have been studied as prognostic factors in various types of cancer, including non-small cell lung carcinoma (NSCLC) $(12,13)$, prostate cancer $(14)$, colorectal carcinoma (15), lymphoma (16) and renal carcinoma (17). The present study examined the expression of these immunohistochemical markers in surgically resected lung carcinoids and their association with patient survival.

\section{Materials and methods}

Study design. Patients that had been subjected to thoracic surgery with a histologic diagnosis of a lung carcinoid between March 2005 and March 2019 at the Department of Thoracic Surgery of Athens Medical Group (Athens, Greece), were retrospectively evaluated. The assessment of the patients included their medical history, clinical examination, chest X-ray and computed tomography (CT) of the chest, brain and upper abdomen. In addition, bone scintiscan had been performed. All patients had a preoperative examination with a fiberoptic bronchoscope, and in certain patients, endoscopic biopsy was performed, while CT-guided fine-needle aspiration biopsy was performed for tumors in the lung periphery. All samples that were resected during surgery, including mediastinal and hilar lymph nodes, were assessed by pathological examination. The categorization of the tumors into TCs and ACs was performed as stated by the World Health Organization (18). Estimation of the carcinoid stage was made in accordance with the 8th TNM staging system for lung cancer (19). Age, sex, type of carcinoid, stage and the expression of the immunohistochemical markers CD56, TTF1, synaptophysin, CEA, EMA and NSE were recorded and the association with the patients' survival rates was determined.

Histological examination. The resected lung tissues were fixed in a $10 \%$ neutral buffered formalin solution. The specimens were set in paraffin and sliced for hematoxylin and eosin staining (2- $\mu \mathrm{m}$-thick for each section). Antigen retrieval was performed using EnVision Flex Target Retrieval Solution High pH (Envision Pathology, LLC). The stainings of slides were applied in the AutoStainer Link 48 instrument (DAKO; Agilent Technologies, Inc.). Immunohistochemical staining against CD56, TTF-1, synaptophysin, CEA, NSE and EMA was performed to confirm the diagnosis of lung carcinoids by an experienced pathologist (AP). The samples were incubated with the antibodies and the detection of positive reactions was made usingthe EnVision Detection System (Envision Pathology, LLC). The features of the antibodies and staining protocols used are provided in Table I.

Evaluation of stainings. Immunohistochemistry marker expression was quantified by expression intensity (weak, moderate, strong) and the percentage of immunohistochemistry marker-positive tumor cells in fields of view of 200-fold magnification by an experienced pathologist (AP). Synaptophysin was considered positive if $>90 \%$ of the neoplastic cells exhibited at least moderate staining intensity. TTF-1 was scored positive if $>10 \%$ of the nuclei of tumor cells were positive and the staining intensity was moderate or strong. Immunostaining for NSE, EMA and CEA expression was considered negative if $<5 \%$ of the cells had weak staining intensity and positive if $>5 \%$ of the cells had weak staining intensity or if the cells exhibited moderate and strong staining intensity. Immunostaining for CD56 expression was considered negative if $<10 \%$ of the cells had weak staining intensity and positive if $>10 \%$ of the cells had weak staining intensity or if the cells had moderate and strong staining intensity (20).

Statistical analysis. SPSS version 13.0 (SPSS, Inc.) was used for statistical analysis. The assessment of survival data was performed by Kaplan-Meier analysis and the comparison of survival curves was made using the log-rank test. Survival was estimated in units of months from surgery. The Cox hazard regression model, including relative risk, probability and $95 \%$ confidence interval was also utilized for univariate analysis and multivariate analysis of the prognostic factors and 5\% was selected as the level of statistical significance.

\section{Results}

Clinicopathological and demographic characteristics of the patients. A total of 108 patients hospitalized at the Department of Thoracic Surgery of Athens Medical Group (Athens, Greece), were analyzed in the present study. AC was diagnosed in 28 patients (16 males and 12 females; mean age, 56.1 years) and TC was diagnosed in 80 patients ( 36 males and 44 females; mean age, 47.5 years). Surgical resection was the only treatment that patients received. Furthermore, 80 patients, 18 patients and 10 patients were categorized as stage I, II and III, respectively. Immunohistochemical staining for CD56, TTF-1, synaptophysin, CEA, NSE and EMA was recorded in 102 patients. CD56 expression was noted in 78 specimens (76.5\%), TTF-1 expression in 12 specimens $(11.8 \%)$, synaptophysin expression in 58 specimens $(56.9 \%)$, CEA expression in 14 specimens (13.7\%), and NSE and EMA were detected in $40(39.2 \%)$ and $6(5.9 \%)$ specimens, respectively (Table II). Fig. S1 shows immunohistochemical staining of typical lung carcinoid samples.

Patients' outcome and correlation with immunohistochemistry markers expression. The patients were followed up for a mean duration of 96 months. All of the patients had good attendance of their follow-up appointments. Of the 108 patients, eight died. All of the deaths were related to the carcinoids and two of the 
Table I. Features of the antibodies and staining protocols used for immunohistochemistry.

\begin{tabular}{|c|c|c|c|c|}
\hline Target & Supplier & Clone & Dilution & Incubation time (min) \\
\hline CD56 & Cell Marque & 123C3.D5 & $1 / 200$ & 30 \\
\hline TTF-1 & LEICA & NCL-L-TTF-1 & $1 / 50$ & 30 \\
\hline Synaptophysin & LEICA & NCL-L-SYNAP-299 & $1 / 50$ & 30 \\
\hline CEA & Cell Marque & CEA31 & $1 / 300$ & 20 \\
\hline NSE & DAKO & BBS/NC/VI-H14 & Ready to use & 20 \\
\hline EMA & LEICA & NCL-L-EMA & $1 / 100$ & 20 \\
\hline
\end{tabular}

The detection system was Envision and pretreatment was performed with the Envision Flex Target Retrieval Solution High pH. CEA, carcinoembryonic antigen; EMA, epithelial membrane antigen; NSE, neuron-specific enolase; TTF-1, thyroid transcription factor 1; NCL-L-TTF-1, liquid mouse monoclonal antibody to TTF-1; NCL-L-SYNAP-299, mouse anti-human synaptophysin monoclonal antibody; BBS/NC/VI-H14, mouse monoclonal anti-enolase 2/NSE antibody; NCL-L-EMA, anti-epithelial membrane antigen monoclonal antibody.

Table II. Clinicopathological and demographic characteristics of the patients.

\begin{tabular}{|c|c|c|c|}
\hline Parameter & $\mathrm{TC}$ & $\mathrm{AC}$ & All \\
\hline \multicolumn{4}{|l|}{ Sex } \\
\hline Male & $36(33.3)$ & $16(14.8)$ & $52(48.1)$ \\
\hline Female & $44(40.7)$ & $12(11.1)$ & $56(51.9)$ \\
\hline \multicolumn{4}{|l|}{ Age, years } \\
\hline Mean \pm SD & $47.5 \pm 76.0$ & $56.1 \pm 24.0$ & $51.5 \pm 11.0$ \\
\hline Median (range) & $50.0(11-80)$ & $65.0(15-80)$ & $52.0(11-80)$ \\
\hline \multicolumn{4}{|l|}{ Stage } \\
\hline I & $64(59.3)$ & $16(14.8)$ & $80(74.1)$ \\
\hline II & $14(13.0)$ & $4(3.7)$ & $18(16.7)$ \\
\hline III & $2(1.8)$ & $8(7.4)$ & $10(9.2)$ \\
\hline \multicolumn{4}{|l|}{ TTF-1 } \\
\hline Positive & $6(5.9)$ & $6(5.9)$ & $12(11.8)$ \\
\hline Negative & $70(68.6)$ & $20(19.6)$ & $90(88.2)$ \\
\hline \multicolumn{4}{|l|}{ CD56 } \\
\hline Positive & $56(54.9)$ & $22(21.6)$ & $78(76.5)$ \\
\hline Negative & $20(19.2)$ & $4(4.3)$ & $24(23.5)$ \\
\hline \multicolumn{4}{|l|}{ Synaptophysin } \\
\hline Positive & $44(43.1)$ & $14(13.8)$ & $58(56.9)$ \\
\hline Negative & $32(31.4)$ & $12(11.7)$ & $44(43.1)$ \\
\hline \multicolumn{4}{|l|}{ CEA } \\
\hline Positive & $12(11.7)$ & $2(2.0)$ & $14(13.7)$ \\
\hline Negative & $64(62.7)$ & $24(23.6)$ & $88(86.3)$ \\
\hline \multicolumn{4}{|l|}{ NSE } \\
\hline Positive & $32(31.4)$ & $8(7.8)$ & $40(39.2)$ \\
\hline Negative & $44(43.1)$ & $18(17.7)$ & $62(60.8)$ \\
\hline \multicolumn{4}{|l|}{ EMA } \\
\hline Positive & $2(2.0)$ & $4(3.9)$ & $6(5.9)$ \\
\hline Negative & $74(72.5)$ & $22(21.6)$ & $96(94.1)$ \\
\hline
\end{tabular}

Values are expressed as n (\%) unless otherwise specified. AC, atypical carcinoid; CEA, carcinoembryonic antigen; EMA, epithelial membrane antigen; NSE, neuron-specific enolase; SD, standard deviation; TTF-1, thyroid transcription factor 1; TC, typical carcinoid.

patients were in stage I of the disease, four patients were in stage II and two patients in stage III; furthermore, six patients had AC and two had TC. There was a statistically significant difference in survival between patients with positive staining 
Table III. Kaplan-Meier survival analysis based on immunohistochemical markers.

\begin{tabular}{|c|c|c|c|c|}
\hline Marker/expression status & $\begin{array}{l}\text { Log-rank P-value for the } \\
\text { difference between the } \\
\text { Kaplan-Meier curves }\end{array}$ & Survival time (months) & $95 \% \mathrm{CI}$ & Survival (\%) \\
\hline TTF1 & 0.472 & & & \\
\hline Negative & & 79.0 & $70.0-88.0$ & 100.0 \\
\hline Positive & & 47.0 & $26.2-67.7$ & 89.1 \\
\hline CD56 & 0.869 & & & \\
\hline Negative & & 143.2 & $126.4-160.0$ & 84.6 \\
\hline Positive & & 140.8 & $126.2-155.1$ & 92.3 \\
\hline Synaptophysin & 0.139 & & & \\
\hline Negative & & 133.9 & $119.4-148.6$ & 82.6 \\
\hline Positive & & 155.6 & $145.7-165.5$ & 96.6 \\
\hline CEA & 0.472 & & & \\
\hline Negative & & 80.2 & $71.3-89.2$ & 88.9 \\
\hline Positive & & 43.8 & $23.6-64.0$ & 100.0 \\
\hline NSE & 0.044 & & & \\
\hline Negative & & 150.0 & $137.3-162.7$ & 96.8 \\
\hline Positive & & 137.2 & $122.2-152.3$ & 81.0 \\
\hline EMA & 0.637 & & & \\
\hline Negative & & 76.8 & $68.1-85.5$ & 89.8 \\
\hline Positive & & 51.3 & $5.3-97.3$ & 100.0 \\
\hline
\end{tabular}

CEA, carcinoembryonic antigen; EMA, epithelial membrane antigen; NSE, neuron-specific enolase; TTF-1, thyroid transcription factor 1.

Table IV. Univariate Cox regression analysis of immunohistochemistry markers.

\begin{tabular}{lccc}
\hline Variable & Exp(B) & P-value & $95 \%$ CI for Exp(B) \\
\hline TTF1 (positive vs. negative) & 0.346 & 0.997 & $0.000-3 \times 10^{243}$ \\
CD56 (positive vs. negative) & 854.316 & 0.241 & $0.011-7 \times 10^{7}$ \\
Synaptophysin (positive vs. negative) & 15.535 & 0.342 & $0.054-4432.459$ \\
CEA (positive vs. negative) & 0.346 & 0.997 & $0.000-3 \times 10^{243}$ \\
NSE (positive vs. negative) & 54202.8 & 0.069 & $0.420-7 \times 10^{9}$ \\
EMA (positive vs. negative) & 77251.8 & 0.673 & $0.000-4 \times 10^{27}$ \\
\hline
\end{tabular}

$\operatorname{Exp}(B)$, relative risk between the groups; CEA, carcinoembryonic antigen; EMA, epithelial membrane antigen; NSE, neuron-specific enolase; TTF-1, thyroid transcription factor 1.

for NSE and patients with negative staining for NSE: Patients with expression of NSE had unfavorable survival (137.2 vs. 150.0 months, $\mathrm{P}=0.044)$. The survival rates in relation to the expression of immunohistochemistry markers are presented in Table III. Fig. S2 shows Kaplan-Meier curves.

Univariate and multivariate Cox regression analysis. According to the univariate Cox regression analysis, expression of the immunohistochemistry markers CD56, TTF-1, synaptophysin, CEA, NSE and EMA was not significantly associated with patient survival (Table IV). As indicated by the multivariate Cox regression analysis, NSE was an independent factor influencing survival of patients with $\mathrm{AC}(\mathrm{P}=0.046)$, while the stage was an independent factor for survival in patients with TC $(\mathrm{P}=0.005$; Table V).

\section{Discussion}

According to the results of the present study, among the factors examined, the stage was an independent prognostic factor for patients with TC. The stage of lung carcinoids has been reported as a prognostic factor in several studies. Ramirez et al (21), in a study of 169 patients with lung carcinoids, demonstrated a statistically significant association between tumor stage and survival. Furthermore, Filosso et al (22), in a study of 157 patients with surgically resected pulmonary carcinoids, 
Table V. Multivariate analysis of prognostic factors analyzed by Cox's hazard regression model (forward stepwise).

\begin{tabular}{lc} 
A, Atypical histology & \\
\hline Variable & P-value \\
\hline TTF1 (positive vs. negative) & $\mathrm{NS}$ \\
CD56 (positive vs. negative) & 0.527 \\
Synaptophysin (positive vs. negative) & 0.460 \\
CEA (positive vs. negative) & $\mathrm{NS}$ \\
NSE (positive vs. negative) & 0.046 \\
EMA (positive vs. negative) & $\mathrm{NS}$ \\
Age ( $\geq 45$ vs. <45 years) & 0.157 \\
Sex (male vs. female) & 0.157 \\
Stage & \\
I vs. II & 0.460 \\
I vs. III & 0.460
\end{tabular}

B, Typical histology

\begin{tabular}{lc}
\hline Variable & P-value \\
\hline TTF1 (positive vs. negative) & 0.705 \\
CD56 (positive vs. negative) & 0.527 \\
Synaptophysin (positive vs. negative) & 0.248 \\
CEA (positive vs. negative) & 0.705 \\
NSE (positive vs. negative) & 0.083 \\
EMA (positive vs. negative) & 0.705 \\
Age ( $\geq 45$ vs. <45 years) & 0.394 \\
Sex (male vs. female) & 0.131 \\
Stage & \\
I vs. II & 0.005 \\
I vs. III & 0.005
\end{tabular}

CEA, carcinoembryonic antigen; EMA, epithelial membrane antigen; NSE, neuron-specific enolase; NS, no significance; TTF-1, thyroid transcription factor 1 .

concluded that nodal deposition and advanced stage were associated with unfavorable survival.

Certain studies have explored the prognostic influence of tissue expression of CEA in cancer patients. Wang et al (23) determined in a large series of 183 patients with stage IB NSCLC that tumor expression of CEA was an independent prognostic factor for survival $(\mathrm{P}<0.05)$. In a study from 1986 examining a small series $(n=31)$ of patients with lung carcinoids, tumors with positive immunohistochemical staining for CEA demonstrated more aggressive behavior, based on survival rates and presence of metastases, than those that did not stain for CEA, with a statistically significant difference $(\mathrm{P}=0.01)(9)$. In the present study, no significant association between CEA expression and survival outcomes was obtained for patients with lung carcinoids.

A study by Ianniello et al (24) examining 34 patients with pulmonary carcinoids suggested that TTF-1 may be a prognostic factor, while in a study by Vesterinen et al (20), in a series of 133 patients with lung carcinoids, expression of TTF-1 was not associated with patient outcomes. In the present study, no significant association of TTF-1 expression and patient survival was obtained.

CD56 expression has been investigated as a prognostic factor in patients with SCLC (25) and both CD56 and synaptophysin have been linked to poor prognosis in patients with NSCLC (26). To the best of our knowledge, the present study was the first to evaluate the role of CD56 and synaptophysin tissue expression in the prognosis of lung carcinoids. It was concluded that CD56 and synaptophysin expression were not significantly associated with patient survival.

EMA expression has been described as a prognostic factor for survival in lymphomas, renal carcinoma and hepatocellular carcinoma $(17,27,28)$. The present study was the first to explore the prognostic role of EMA expression in lung carcinoids, to the best of our knowledge, and according to the results, EMA expression was not related to patients' survival outcomes.

Serum NSE levels have been described as a prognostic factor for the outcome of patients with SCLC (29) and NSLC (30,31). Tissue expression of NSE has been associated with longer survival in patients with advanced NSCLC in a study by Petrovic et al (32), while in a study by Tiseo et al (33), NSE expression rendered NSCLC patients with poor prognosis. The present study is one of the largest to date to examine the influence of NSE expression on the survival of patients with pulmonary carcinoids. The results indicated that patients with NSE expression had unfavorable survival in comparison to patients without NSE expression in their lung carcinoid specimens and NSE was an independent prognostic factor for survival of patients with AC.

The strength of the present study lies in the large number of patients, accompanied by reliable follow-up and outcome data. However, the current study also has certain limitations. Despite the large number of participants, the group of patients with AC was relatively smaller, which may have caused bias in the multivariate analysis. Furthermore, it is a single-center study with a retrospective design and has an inferior level of evidence compared to prospective studies. Retrospective cohort studies require large sample sizes, particularly if outcomes are rare. Another limitation is the lack of a control group, which may make it impossible to draw meaningful conclusions from a study. Larger multicenter, prospective studies are required to provide more detailed results.

In conclusion, the present study was the first, to the best of our knowledge, to study the impact of the expression of the immunohistochemical markers CD56, synaptophysin and EMA on the survival of patients with surgically resected lung carcinoids and is one of a handful of studies exploring the role of the expression of TTF-1, NSE and CEA in the prognosis of such patients. There was a statistically significant difference in survival rates of patients with positive staining for NSE compared to that of patients with negative staining for NSE. In addition, NSE was an independent influencing factor of survival for patients with AC, while the stage was an independent factor of survival for patients with TC. Further studies are required to assess the effect of the expression of these immunohistochemical markers on the survival of patients with lung carcinoids. 


\section{Acknowledgements}

Not applicable.

\section{Funding}

No funding was received.

\section{Availability of data and materials}

The datasets used and/or analyzed during the current study are available from the corresponding author upon reasonable request.

\section{Authors' contributions}

JD, JJ and EZ conceptualized the study. AP performed the immunohistochemical examination and prepared Table I. VEG, CN, NG, CD and SC wrote and prepared the draft of the manuscript and were substantial contributions to the conception or design of the work. ED and DAS provided critical revisions and made the final approval of the version to be published. NT and PS performed the statistical analysis. PP, AAF and AG prepared the other tables. VEG and EZ confirm the authenticity of all the raw data. All authors contributed to the revision of the manuscript and read and approved the final version of the manuscript.

\section{Ethics approval and consent to participate}

Ethical approval was obtained from the Research Ethics Committee of Athens Medical Group (Athens, Greece; file accession no. 4234). The study was in line with the declaration of Helsinki from 1995 (and its revision in Edinburgh 2000). Written informed consent was obtained from the patients for the entry of their data into a database and use for research.

\section{Patient consent for publication}

Not applicable.

\section{Competing interests}

The authors declare that they have no competing interests.

\section{References}

1. Gosain R, Mukherjee S, Yendamuri SS and Iyer R: Management of typical and atypical pulmonary carcinoids based on different established guidelines. Cancers (Basel) 10: 510, 2018.

2. Rosado de Christenson ML, Abbott GF, Kirejczyk WM, Galvin JR and Travis WD: Thoracic carcinoids: Radiologic-pathologic correlation. Radiographics 19: 707-736, 1999.

3. Hemminki $\mathrm{K}$ and $\mathrm{Li} \mathrm{X}$ : Incidence trends and risk factors of carcinoid tumors: A nationwide epidemiologic study from Sweden. Cancer 92: 2204-2210, 2001.

4. Quaedvlieg PF, Visser O, Lamers CB, Janssen-Heijen ML and Taal BG: Epidemiology and survival in patients with carcinoid disease in The Netherlands. An epidemiological study with 2391 patients. Ann Oncol 12: 1295-1300, 2001.

5. Rekhtman N: Neuroendocrine tumors of the lung: An update. Arch Pathol Lab Med 134: 1628-1638, 2010.
6. Zeng Y, Zhu Y, Ding Y, Xu L, Zhai B, Zhang X, Ge Q, Li J, Song Q, Li X and Zhang Z: Analysis of lung biopsies using the 2015 WHO criteria and detection of sensitizing mutations-a single-institution experience of 5032 cases. Diagn Pathol 15: 59 , 2020.

7. Mjønes P, Sagatun L, Nordrum IS and Waldum HL: Neuron-specific enolase as an immunohistochemical marker is better than its reputation. J Histochem Cytochem 65: 687-703, 2017.

8. Gledhill A, Sparham C and Dixon MF: Expression of epithelial membrane antigen by carcinoid tumours. J Clin Pathol 43: 696-697, 1990

9. Bishopric GA Jr and Ordóñez NG: Carcinoembryonic antigen in primary carcinoid tumors of the lung. Cancer 58: 1316-1320, 1986.

10. Huang Y, Yang X, Lu T, Li M, Zhao M, Yang X, Ma K, Wang S, Zhan C, Liu Y and Wang Q: Assessment of the prognostic factors in patients with pulmonary carcinoid tumor: A population-based study. Cancer Med 7: 2434-2441, 2018.

11. Georgakopoulou VE, Zygouris E, Nikokiris C, Damaskos C, Pierrakou A, Garmpis N, Garmpi A, Sklapani P, Aravantinou A, Trakas N, et al: Predictive indicators of survival in patients with surgically resected lung carcinoid tumors at a Greek medical center. Cureus 12: e10300, 2020.

12. Graziano SL, Tatum AH, Newman NB, Oler A, Kohman LJ, Veit LJ, Gamble GP, Coleman MJ, Barmada S and O'Lear S: The prognostic significance of neuroendocrine markers and carcinoembryonic antigen in patients with resected stage I and II non-small cell lung cancer. Cancer Res 54: 2908-2913, 1994.

13. Yaman B, Nart D, Ekren PK, Çok G and Veral A: Expression of p63, TTF-1 and maspin in non-small cell lung carcinoma and their effect on the prognosis and differential diagnosis. Turk Patoloji Derg 31: 163-174, 2015.

14. Ather MH, Abbas F, Faruqui N, Israr M and Pervez S: Correlation of three immunohistochemically detected markers of neuroendocrine differentiation with clinical predictors of disease progression in prostate cancer. BMC Urol 8: 21, 2008.

15. Tudoraşcu DR, Pirici D, Târtea EA, Mustafa ER, Florescu C, Vere CC, Balea AM, Puiu I, Târtea GC and Albu VC: Synaptophysin expression as prognostic factor for survival in colorectal carcinomas. Rom J Morphol Embryol 58: 1409-1415, 2017.

16. Wang L, Wang Z, Xia ZJ, Lu Y, Huang HQ and Zhang YJ: CD56-negative extranodal NK/T cell lymphoma should be regarded as a distinct subtype with poor prognosis. Tumour Biol 36: 7717-7723, 2015.

17. Bamias A, Chorti M, Deliveliotis C, Trakas N, Skolarikos A Protogerou B, Legaki S, Tsakalou G, Tamvakis $\mathrm{N}$ and Dimopoulos MA: Prognostic significance of CA 125, CD44, and epithelial membrane antigen in renal cell carcinoma. Urology 62: 368-373, 2003.

18. Travis WD, Brambilla E, Nicholson AG, Yatabe Y, Austin JHM, Beasley MB, Chirieac LR, Dacic S, Duhig E, Flieder DB, et al: The 2015 World Health Organization classification of lung tumors: Impact of genetic, clinical and radiologic advances since the 2004 classification. J Thorac Oncol 10: 1243-1260, 2015.

19. Feng SH and Yang ST: The new 8th TNM staging system of lung cancer and its potential imaging interpretation pitfalls and limitations with CT image demonstrations. Diagn Interv Radiol 25: 270-279, 2019.

20. Vesterinen T, Mononen S, Salmenkivi K, Mustonen H, Räsänen J, Salo JA, Ilonen I, Knuuttila A, Haglund C and Arola J: Clinicopathological indicators of survival among patients with pulmonary carcinoid tumor. Acta Oncol 57: 1109-1116, 2018.

21. Ramirez RA, Beyer DT, Diebold AE, Voros BA, Chester MM, Wang YZ, Boudreaux JP, Woltering EA, Uhlhorn AP, Ryan $\mathrm{P}$, et al: Prognostic factors in typical and atypical pulmonary carcinoids. Ochsner J 17: 335-340, 2017.

22. Filosso PL, Ruffini E, Di Gangi S, Guerrera F, Bora G, Ciccone G, Galassi C, Solidoro P, Lyberis P, Oliaro A and Sandri A: Prognostic factors in neuroendocrine tumours of the lung: A single-centre experience. Eur J Cardiothorac Surg 45: 521-526, 2014.

23. Wang J, Ma Y, Zhu ZH, Situ DR, Hu Y and Rong TH: Expression and prognostic relevance of tumor carcinoembryonic antigen in stage IB non-small cell lung cancer. J Thorac Dis 4: 490-496, 2012 . 
24. Ianniello A, Sansovini M, Severi S, Nicolini S, Grana CM, Massri K, Bongiovanni A, Antonuzzo L, Di Iorio V, Sarnelli A, et al: Peptide receptor radionuclide therapy with (177) Lu-DOTATATE in advanced bronchial carcinoids: Prognostic role of thyroid transcription factor 1 and (18)F-FDG PET. Eur J Nucl Med Mol Imaging 43: 1040-1046, 2016.

25. Wang X, Zhang Y, Hu M, Wang R, Liu L, Qian K, Li Y and Zhi X: Prognostic and predictive value of thyroid transcription factor-1, CD56, P40 and other clinical characteristics in small cell lung cancer patients. Zhongguo Fei Ai Za Zhi 20: 522-527, 2017 (In Chinese).

26. Feng J, Sheng H, Zhu C, Qian X, Wan D, Su D, Chen X and Zhu L: Correlation of neuroendocrine features with prognosis of non-small cell lung cancer. Oncotarget 7: 71727-71736, 2016.

27. Touhami H, Sotto JJ, Sotto MF, Couderc P, Keddari E, Brahimi S and Kubisz P: Prognostic value of the epithelial membrane antigen (EMA) in the case of Hodgkin's disease. Neoplasma 38: 433-437, 1991

28. Kim GJ, Rhee H, Yoo JE, Ko JE, Lee JS, Kim H, Choi JS and Park YN: Increased expression of CCN2, epithelial membrane antigen, and fibroblast activation protein in hepatocellular carcinoma with fibrous stroma showing aggressive behavior. PLoS One 9: e105094, 2014.
29. Jørgensen LG, Osterlind K, Genollá J, Gomm SA, Hernández JR, Johnson PW, Løber J, Splinter TA and Szturmowicz M: Serum neuron-specific enolase (S-NSE) and the prognosis in small-cell lung cancer (SCLC): A combined multivariable analysis on data from nine centres. Br J Cancer 74: 463-467, 1996.

30. Barlési F, Gimenez C, Torre JP, Doddoli C, Mancini J, Greillier L, Roux F and Kleisbauer JP: Prognostic value of combination of Cyfra 21-1, CEA and NSE in patients with advanced non-small cell lung cancer. Respir Med 98: 357-362, 2004.

31. Jiang R, Wang X and Li K: Predictive and prognostic value of preoperative serum tumor markers is EGFR mutation-specific in resectable non-small-cell lung cancer. Oncotarget 7: 26823-26836, 2016.

32. Petrovic M, Tomic I, Plavec G, Ilic S, Ilic N and Baskic D: Neuron specific enolase tissue expression as a prognostic factor in advanced non small cell lung cancer. J BUON 13: 93-96, 2008.

33. Tiseo M, Ardizzoni A, Cafferata MA, Loprevite M, Chiaramondia M, Filiberti R, Marroni P, Grossi F and Paganuzzi M: Predictive and prognostic significance of neuron-specific enolase (NSE) in non-small cell lung cancer. Anticancer Res 28: 507-513, 2008.

(i) (9) This work is licensed under a Creative Commons

EY No ND Attribution-NonCommercial-NoDerivatives 4.0 International (CC BY-NC-ND 4.0) License. 